\title{
Aplicação da metaheurística GRASP na programação de caminhões para o transporte de aves domésticas
}

\section{Application of the GRASP of trucks in the programming to carry poultry}

\author{
Carlos Ropelatto Fernandes ${ }^{1}$; Celso Carnieri²; Sebastião Geraldo Barbosa ${ }^{3}$
}

\section{Resumo}

O presente trabalho tem como objetivo principal minimizar a média ponderada dos tempos de espera dos caminhões carregados, tendo como peso o número de aves transportadas em cada carga, de forma que esse tempo fique o mais próximo de 25 minutos, que é o tempo considerado ideal pela empresa para a espera do abate das aves. A empresa Avícola trabalha no sistema integrado, contando com cerca de 390 granjas espalhadas ao redor de 38 municípios, que dela distam entre $8 \mathrm{~km}$ a $109 \mathrm{~km}$. O abate diário médio é de 90.000 aves, provenientes de três a doze granjas. O transporte das aves até o abatedouro é feito por uma empresa terceirizada, que conta com uma frota de 13 caminhões com capacidades diferenciadas; o limite da frota é mais ou menos a metade do abate diário, sendo então necessária mais de uma viagem por caminhão. O problema consiste em fazer o roteamento dos caminhões até as granjas e a programação dos horários, satisfazendo a demanda de abate e minimizando o tempo de espera. Como se trata de um problema classificado na literatura como NP-hard, este trabalho propõe o uso de um método heurístico baseado na metaheurística GRASP a qual busca determinar uma solução ótima ou quase ótima para o problema.

Palavras-chave: Metaheurística. GRASP. Roteamento. Programação. Sistema Integrado.

\begin{abstract}
This paper's main objective is to minimize the weighted average waiting time of trucks loaded with weight as the number of birds on each charge carriers, so that time is as close to 25 minutes, what time is considered ideal the company expects to slaughter the birds. The company works on poultry integrated system, with about 390 farms scattered around the 38 cities that are distant from her $8 \mathrm{~km}$ to $109 \mathrm{~km}$. The average daily slaughter of 90,000 birds is from three to twelve farms. The transportation of poultry to the slaughterhouse is done by an outside company, which has a fleet is about half the daily slaughter, was therefore required more than one trip per truck. The problem is to do the routing of trucks to the farms and set the time, satisfying the demand for slaughter and minimizing downtime. As this is a problem in the literature classified as NP-hard, this paper proposes the use of a heuristic method based on GRASP metaheuristic which seeks to determine an optimal solution to the problem.
\end{abstract}

Keywords: Metaheuristic. GRASP. Routing. Programming. Integrated System.

1 Professor Mestre do Colegiado de Matemática, Universidade Estadual do Paraná, Campus de Paranavaí, Avenida Gabriel Esperidião, $\mathrm{s} / \mathrm{n}^{\circ}$, Paranavaí, Paraná, Brasil.

2 Professor Doutor do Programa de Pós-Graduação em Métodos Numéricos em Engenharia, Universidade Federal do Paraná, edifício LAME/CESEC do Centro Politécnico da UFPR, Curitiba, Paraná, Brasil.

3 Professor Mestre do Colegiado de Matemática, Universidade Estadual do Paraná, Campus de Paranavaí, Avenida Gabriel Esperidião, $\mathrm{s} / \mathrm{n}^{\circ}$, Paranavaí, Paraná, Brasil. 


\section{Introdução}

Nas últimas décadas tem-se percebido uma mudança nos hábitos alimentares, principalmente no que diz respeito ao consumo de carnes. Cuidados com a saúde fizeram com que as pessoas passassem a consumir mais carnes brancas, (OLIVO, 2004).

A avicultura brasileira, e também a mundial, se desenvolveu e se modernizou rapidamente, e alcançou níveis elevados de produtividade nos últimos 30 anos. Em 1970, eram necessários 70 dias para o crescimento e engorda de um frango de corte que consumia cerca de $2,0 \mathrm{~kg}$ de ração para 1,0 $\mathrm{kg}$ de ganho de peso, sendo que $80 \%$ do peso vivo poderiam ser considerados comestíveis. Hoje em dia, um frango de corte fica pronto para o abate com 2,4 $\mathrm{kg}$, aos 42 dias, tendo uma conversão alimentar de $1,8 \mathrm{~kg}$ de ração para cada quilograma de ganho de peso. (FERNANDES, 2005).

No agronegócio brasileiro, a avicultura industrial destacou-se nas últimas décadas por uma trajetória de incremento tecnológico expressivo, alavancada pela destacada articulação entre os diferentes agentes que o compõem. Assim, a cadeia produtiva de aves de corte assegura ao país posição de destaque no cenário mundial, ocupando o segundo lugar na produção mundial de carne de frango.

Atualmente a grande preocupação é desenvolver projeto que consiga minimizar o tempo de espera e o tempo de transporte das aves dos barracões até o local do abatedouro. O presente projeto procurou desenvolver um método heurístico, baseado na metaheurística GRASP, para o roteamento e a programação dos horários dos caminhões que realizam o transporte das aves para o abate, fazendo com que a média ponderada do tempo de espera das aves, desde a chegada na Avícola até o momento do abate, fique o mais próximo de 25 minutos. (FERNANDES, 2005).

Através deste projeto procurou-se também:

- Equilibrar a carga de trabalho entre os motoristas, quanto à distância percorrida;
- Equilibrar a carga de trabalho entre as equipes de apanha, quanto à quantidade de aves apanhadas;

- Diminuir a ociosidade dos motoristas, quanto ao tempo de espera;

- Diminuir a perda de peso e a taxa de mortalidade das aves.

A otimização do processo de roteirização e de programação dos horários dos caminhões, através da implementação computacional do método heurístico proposto, produzirá alguns benefícios, tais como: diminuição da taxa de mortalidade; diminuição da perda de peso das aves; aumento do número de aves abatidas, gerando lucro tanto para empresa quanto para os produtores; redução do número de horas trabalhadas pelos motoristas; ganho de tempo, por parte do responsável pela roteirização e programação dos horários dos caminhões, para a realização de outras atividades. Também beneficiará um grande número de agentes envolvidos no processo, tais como: a empresa de transporte das aves, a avícola, os produtores, os motoristas e as equipes de apanha.

\section{Metaheurísticas}

Metaheurísticas são procedimentos destinados a encontrar uma boa solução, eventualmente a ótima, constituindo na aplicação, em cada passo, de uma heurística subordinada, a qual tem que ser modelada para cada problema específico. A principal característica das metaheurísticas é a capacidade que estas possuem de escapar de ótimos locais (GOMES; DINIZ; MARTINHON, 2000).

Pode-se destacar algumas metaheurísticas, como: GRASP, Busca Tabu, VNS (Variable Neighborhood Search) e VND (Variable Neighborhood Descemt). Estes dois últimos são métodos de busca local, que foram proposto por Hansen e Mladenović (1997).

\section{GRASP}

O problema de roteamento e programação 
de veículos pertence a uma classe de problemas denominados Problemas de Otimização Combinatória.

A metaheurística GRASP (FEO; RESENDE, 1989, 1995), pode ser vista como um processo iterativo caracterizado por partidas múltiplas onde cada iteração consiste de duas fases:

- $\mathrm{Na}$ primeira fase, chamada fase de construção, uma solução inicial $s$ é gerada, elemento a elemento;

- Na segunda fase, denominada fase de busca local, um ótimo local na vizinhança, $\operatorname{Viz}(s)$, da solução construída é pesquisado.

A ideia principal da metaheurística GRASP baseia-se na utilização de diferentes soluções iniciais como ponto de partida para a busca local (AIEX, 2004).

FESTA e RESENDE (2002) fornecem uma extensa literatura sobre a metaheurística GRASP.

Um pseudo-código do GRASP é dado na Figura 1. A linha 1 do pseudo-código corresponde aos dados de entrada do problema. As iterações GRASP têm lugar nas linhas 2 a 6 , e terminam quando o número máximo ocorre ou quando a solução procurada encontrada é satisfeita. Na linha 3 tem-se a fase de construção e na linha 4 a fase de busca local. Se uma melhoria na solução for encontrada, a atualização ocorre na linha 5. E por último, na linha 7, a melhor solução é retornada como resultado.

Figura 1 - Um pseudo-Código Genérico do GRASP.

\begin{tabular}{|ll|}
\hline \multicolumn{3}{|l|}{ Procedimento GRASP( ); } \\
1 & DadosEntrada( ); \\
2 & Enquanto critério de parada não for \\
satisfeito, faça: & \\
3 & ConstSolInicGulosaAleatória(s) \\
4 & BuscaLocal(s, Viz(s)); \\
5 & AtualizSol(s, melhorSolEnc); \\
6 & Fim Enquanto; \\
7 & Retorna(melhorSolEnc); \\
Fim GRASP.
\end{tabular}

O número máximo de iterações é o critério de parada utilizado na maioria dos problemas. Porém, podem ser definidos outros critérios, como por exemplo, se a solução procurada encontrada for satisfeita ou até mesmo determinar um tempo máximo de execução do algoritmo.

\section{Fase Construtiva}

Nesta fase, uma solução viável é construída iterativamente, inserindo-se na solução parcial um elemento de cada vez. A cada iteração da fase construtiva são avaliados somente elementos que podem ser adicionados à solução sem violar as restrições de viabilidade; esses elementos são chamados de elementos candidatos. O próximo elemento a ser adicionado à solução é determinado ordenando-se todos os elementos candidatos em uma lista chamada de lista de candidatos. Então, é formada uma lista que contém os melhores candidatos, a qual recebe o nome de Lista Restrita de Candidatos (LRC).

\section{Fase de Busca Local}

A solução inicial $s$ gerada pela fase construtiva não tem garantia de ser localmente ótima. Portanto, após a construção da solução inicial s, faz-se uma busca na estrutura de vizinhança Viz(s) relativa à essa solução. Esse procedimento realiza sucessivas trocas da solução corrente, sempre que uma melhor solução é encontrada na $\operatorname{Viz}(s)$. O procedimento termina quando nenhuma solução melhor é encontrada.

\section{Materiais e Métodos}

O Método Heurístico Proposto GRASP Aplicado ao Problema de Espera

O GRASP, foi proposto por Feo e Resende (1995), trata-se de um método iterativo, o qual é constituído de duas fases: 
- Na primeira fase, chamada fase construtiva, uma solução inicial, Sol_Ini, é gerada, elemento a elemento;

- $\quad \mathrm{Na}$ segunda fase, denominada fase de busca local, um ótimo local na vizinhança da solução construída é pesquisado.

A melhor solução é retornada como resultado.

Cálculo do tempo de viagem vazio (tvv) e do tempo de viagem carregado (tvc)

Para o cálculo do $t v v$ e do $t v c$, procedeu-se da maneira descrita abaixo, onde se calculou primeiro o tvc.

1. Tomaram-se os horários de términos dos carregamentos de cada caminhão nas respectivas granjas, conforme Tabela 1 .

2. Tomaram-se os horários de chegada de cada caminhão na Avícola;

3. Subtraiu-se cada um dos valores encontrados no item 2, dos encontrados no item 1; para os respectivos caminhões, vindos das respectivas granjas;

4. Feito isso, obteve-se a média aritmética do tvc para cada granja;

5. Para o cálculo do $t v v$, após pesquisa feita com os motoristas, verificou-se que esse valor correspondia a aproximadamente $80 \%$ do valor do $t v c$, de cada granja, valor este adotado para os cálculos;

6. Para finalizar, construiu-se a Tabela 2, contendo a relação de todas as granjas atendidas, para o abate, pela Avícola, levando em consideração os relatórios das outras duas equipes de apanha, B e C.

Tabela 1 - Relatório das atividades da equipe de apanha A

\begin{tabular}{lllllllll}
\hline Granjeiros & Cidade & Placa & Início & A.cx. & Placa & Início & Término & Aves \\
\hline Produtor 1 & São Tomé & ACO & $23: 00$ & 8 & HQG & $23: 00$ & $23: 54$ & 2.816 \\
Produtor 1 & São Tomé & KDO & $23: 40$ & 8 & KDO & $23: 54$ & $05: 50$ & 3.456 \\
Produtor 1 & São Tomé & AEY & $00: 30$ & 8 & AEY & $00: 50$ & $01: 48$ & 3.456 \\
Produtor 1 & São Tomé & BWP & $01: 20$ & 8 & BWP & $01: 48$ & $03: 00$ & 3.456 \\
Produtor 1 & São Tomé & JTP & $02: 10$ & 8 & JTP & $03: 00$ & $04: 13$ & 3.456 \\
Produtor 1 & São Tomé & HQG & $03: 00$ & 8 & HQG & $04: 13$ & $04: 48$ & 2.400 \\
Produtor 1 & São Tomé & AEA & $03: 40$ & 8 & AEA & $04: 48$ & $05: 28$ & 2.144 \\
Produtor 2 & São Carlos & KOL & $05: 30$ & 8 & KOL & $06: 20$ & $07: 13$ & 3.456 \\
Produtor 2 & São Carlos & ACJ & $06: 20$ & 8 & ACJ & $07: 13$ & $08: 06$ & 3.160 \\
Produtor 2 & São Carlos & KDO & $07: 10$ & 8 & KDO & $08: 06$ & $09: 06$ & 3.456 \\
Produtor 2 & São Carlos & BWP & $08: 00$ & 8 & BWP & $09: 06$ & $09: 57$ & 3.027 \\
\hline
\end{tabular}

Fonte: Trevizoli e Cia Ltda 
Tabela 2 - Demonstrativo de abate diário por integrado da AVÍCOLA FELIPE

\begin{tabular}{|c|c|c|c|c|c|c|c|c|c|c|}
\hline Carga & Integrado & Placa & $\begin{array}{c}\text { Quant. } \\
\text { Aves }\end{array}$ & $\begin{array}{c}\text { Peso } \\
\text { Líquido }\end{array}$ & $\begin{array}{l}\text { Peso } \\
\text { Médio }\end{array}$ & $\begin{array}{l}\text { Morta- } \\
\text { lidade }\end{array}$ & $\begin{array}{l}\% \text { de } \\
\text { Mort. }\end{array}$ & $\begin{array}{l}\text { Hor. } \\
\text { Cheg. }\end{array}$ & $\begin{array}{l}\text { Hor. } \\
\text { Abate }\end{array}$ & $\begin{array}{l}\text { Tempo } \\
\text { Espera }\end{array}$ \\
\hline 1 & Produtor 1 & $\mathrm{ACO}$ & 2.816 & 6.500 & 2,308 & 3 & 0,11 & $01: 45$ & $05: 00$ & $3: 15$ \\
\hline 2 & Produtor 1 & $\mathrm{KDO}$ & 3.456 & 7.940 & 2,297 & 1 & 0,03 & $02: 30$ & $05: 22$ & $2: 52$ \\
\hline 3 & Produtor 1 & AEY & 3.456 & 8.060 & 2,332 & 1 & 0,03 & $03: 40$ & $05: 51$ & $2: 11$ \\
\hline 4 & Produtor 1 & BWP & 3.456 & 7.790 & 2,254 & 2 & 0,06 & $04: 36$ & $06: 19$ & $1: 43$ \\
\hline 5 & Produtor 1 & JTP & 3.456 & 7.680 & 2,222 & 1 & 0,03 & $05: 48$ & $06: 47$ & $0: 59$ \\
\hline 6 & Produtor 1 & HQG & 2.400 & 5.330 & 2,221 & 0 & 0,00 & $06: 53$ & $07: 15$ & $0: 22$ \\
\hline 7 & Produtor 1 & AEA & 2.144 & 4.550 & 2,122 & 1 & 0,03 & $07: 20$ & $07: 35$ & $0: 15$ \\
\hline 8 & Produtor 5 & $\mathrm{BXB}$ & 4.456 & 8.790 & 2,543 & 2 & 0,06 & $07: 22$ & $07: 54$ & $0: 32$ \\
\hline 9 & Produtor 5 & GQQ & 2.816 & 7.200 & 2,557 & 1 & 0,03 & $07: 48$ & 08:20 & $0: 32$ \\
\hline 10 & Produtor 2 & KOL & 3.456 & 8.580 & 2,483 & 2 & 0,06 & $08: 24$ & $08: 46$ & $0: 22$ \\
\hline 11 & Produtor 2 & ACJ & 3.160 & 7.970 & 2,552 & 0 & 0,00 & 09:05 & $09: 34$ & $0: 29$ \\
\hline 12 & Produtor 5 & $\mathrm{ACO}$ & 2.816 & 7.070 & 2,511 & 8 & 0,28 & 09:03 & $10: 26$ & $1: 23$ \\
\hline 13 & Produtor 5 & AeY & 3.456 & 8.850 & 2,561 & 3 & 0,11 & 09:49 & $11: 06$ & $1: 17$ \\
\hline 14 & Produtor 2 & $\mathrm{KDO}$ & 3.456 & 8.730 & 2,526 & 3 & 0,11 & $10: 10$ & $11: 34$ & $1: 24$ \\
\hline 15 & Produtor 5 & JTP & 2.026 & 5.040 & 2,488 & 4 & 0,20 & $10: 16$ & $12: 02$ & $1: 46$ \\
\hline 16 & Produtor 2 & BWP & 3.027 & 7.390 & 2,441 & 3 & 0,11 & $10: 50$ & $12: 19$ & $1: 29$ \\
\hline 17 & Produtor 6 & GQQ & 2.464 & 5.840 & 2,370 & 11 & 0,45 & $11: 49$ & $12: 44$ & $0: 55$ \\
\hline 18 & Produtor 3 & HQG & 2.560 & 6.320 & 2,496 & 1 & 0,03 & $12: 38$ & $13: 02$ & $0: 24$ \\
\hline 19 & Produtor 6 & ACJ & 3.024 & 7.140 & 2,361 & 1 & 0,03 & $12: 53$ & $13: 44$ & $0: 51$ \\
\hline 20 & Produtor 3 & AEA & 2.560 & 6.370 & 2,488 & 3 & 0,11 & $13: 41$ & $14: 20$ & $0: 39$ \\
\hline 21 & Produtor 6 & AEY & 1.917 & 4.490 & 2,342 & 1 & 0,03 & $13: 34$ & $14: 50$ & $1: 16$ \\
\hline 22 & Produtor 3 & $\mathrm{BXB}$ & 3.456 & 8.640 & 2,500 & 2 & 0,06 & $14: 40$ & $15: 13$ & $0: 33$ \\
\hline 23 & Produtor 3 & KOL & 3.267 & 8.170 & 2,501 & 0 & 0,00 & $15: 30$ & $15: 55$ & $0: 25$ \\
\hline 24 & Produtor 4 & $\mathrm{ACO}$ & 2.112 & 4.620 & 2,118 & 3 & 0,11 & $16: 46$ & $16: 55$ & 0:09 \\
\hline 25 & Produtor 4 & $\mathrm{KDO}$ & 2.592 & 5.690 & 2,234 & 0 & 0,00 & $17: 27$ & $18: 27$ & 1:00 \\
\hline 26 & Produtor 4 & JTP & 3.024 & 6.530 & 2,159 & 1 & 0,03 & $18: 28$ & $18: 57$ & $0: 29$ \\
\hline 27 & Produtor 4 & BWP & 3.024 & 6.620 & 2,189 & 0 & 0,00 & $19: 29$ & $19: 36$ & 0:07 \\
\hline 28 & Produtor 4 & HQG & 2.240 & 4.930 & 2,201 & 2 & 0,06 & $20: 22$ & $20: 32$ & $0: 10$ \\
\hline 29 & Produtor 4 & GQQ & 2.464 & 5.400 & 2,192 & 0 & 0,00 & $21: 07$ & $21: 16$ & 0:09 \\
\hline \multirow[t]{2}{*}{30} & Produtor 4 & AEA & 2.128 & 4.660 & 2,190 & 0 & 0,00 & $22: 04$ & $22: 12$ & 0:08 \\
\hline & & Total & 85.685 & 202.990 & 2,369 & 60 & 0,07 & & & \\
\hline
\end{tabular}

Fonte: Trevizoli e Cia Ltda 
Para exemplificar o cálculo do tvc para uma ver Tabela 3. determinada granja foi considerado o Produtor 1,

Tabela 3 - Relatório das atividades da equipe de apanha A

\begin{tabular}{llccccccc}
\hline Granjeiros & Cidade & Dis & Eqa & Cam & Aves & Saída & Chegada & tvcc \\
\hline Produtor 1 & São Tomé & 95 & B & ACO & 2.816 & $23: 54$ & $01: 45$ & $1: 51$ \\
Produtor 1 & São Tomé & 95 & B & KDO & 3.456 & $00: 50$ & $02: 30$ & $1: 40$ \\
Produtor 1 & São Tomé & 95 & B & AEY & 3.456 & $01: 48$ & $03: 40$ & $1: 52$ \\
Produtor 1 & São Tomé & 95 & B & BWP & 3.456 & $03: 00$ & $04: 36$ & $1: 36$ \\
Produtor 1 & São Tomé & 95 & B & JTP & 3.456 & $04: 13$ & $05: 48$ & $1: 35$ \\
Produtor 1 & São Tomé & 95 & B & HQG & 2.400 & $04: 48$ & $06: 53$ & $2: 05$ \\
Produtor 1 & São Tomé & 95 & B & AEA & 2.144 & $05: 28$ & $07: 20$ & $1: 52$ \\
\hline
\end{tabular}

Fonte: Trevizoli e Cia Ltda

1. Primeiramente, calcula-se a diferença entre os horários de chegada, na Avícola, e os respectivos horários de saída, da granja, de cada caminhão;

2. Em seguida, calcula-se a média aritmética dos valores obtidos no item anterior.

\section{Função Objetivo}

Para o Problema de Espera, a função objetivo consiste em minimizar $W$, dada pela Equação 1 a seguir, a qual é definida pelo valor absoluto da diferença entre o tempo de $25 \mathrm{~min}^{4}$ e o quociente entre o somatório do produto da quantidade de aves transportada em cada carga $\left(q a c_{k}\right)$ pelo tempo de espera carregado $\left(t e c_{k}\right)$, e o somatório da quantidade de aves abatidas $\left(q a c_{k}\right)$. Quanto mais próximo de zero estiver $W$, melhor será o tec.

\section{Equação 1}

$\operatorname{Min} W=a b s\left(\frac{\sum_{i=1}^{k}\left(q a c_{i} \cdot t e c_{i}\right)}{\sum_{i=1}^{k} q a c_{i}}-25\right)$
Fase Construtiva - Geração de uma Solução Inicial

Para o Problema de Espera, uma solução inicial viável, Sol_Ini, deve satisfazer as seguintes restrições de viabilidade:

1. Um caminhão, $\operatorname{cam}_{k}$, só pode ser despachado $^{5}$ para uma $s$-ésima carga, $c a r$, se o mesmo já tenha terminado sua $r$-ésima carga, $c a r$; isto é, se $h s a_{s}$ (horário de saída da avícola referente a $s$-ésima carga) $>h t a_{r}$ (horário de término de abate da $r$-ésima carga), $\operatorname{com} s>r$;

2. Um caminhão, $c_{a m}$, poderá transportar, no máximo, até $10 \%$ mais de sua capacidade;

3. Uma equipe de apanha, eqa , não poderá atender mais de uma granja ao mesmo tempo;

4. Quando uma equipe terminar o apanhe na granja $r$ e for iniciar o apanhe na granja $s$, o tempo de deslocamento não deve ser inferior a $40 \mathrm{~min}$, tempo este, considerado necessário para o deslocamento entre as granjas, com $s>r$;

4 Este valor é considerado pela Avícola, como sendo o tempo ideal para que as aves esperem para serem abatidas.

5 Consiste em determinar a granja de apanha e o horário de início do carregamento. 
5. Um motorista não poderá trabalhar mais de 12 horas consecutivas;

6. Um caminhão não poderá começar o carregamento em uma granja $g$ antes que um outro caminhão já tenha terminado de carregar;

7. Um caminhão não poderá chegar ao abatedouro após o horário previsto para o início do abate de sua carga;

8. O tempo mínimo de espera para o abate não deverá ser inferior a 5 min;

9. O tempo máximo de espera para o abate não deverá ser superior a 60 min (esta restrição é aplicada somente na solução final).

Tanto a Solução Inicial, Sol_Ini; quanto a Solução Final, Sol_Fin, serão triplas ordenadas do tipo:

Sol_Fin $=\left\{\left(\text { cam }_{c}, \text { grj }_{g} \text { eqa }\right)_{k}\right\}$, com $k=1, \ldots$, car; $c=1, \ldots, 13 ; g=1, \ldots, n g ; n g=3, \ldots, 12 ; e$ $=1, \ldots, 4$. Onde $c a m_{c}$ é o caminhão $c, g r j$, é a granja $g, e q a_{e}$ é a equipe de apanha $e$ sendo todos referentes ao carregamento da $k$-ésima carga para abate; car é a quantidade de carga e $n g$ é o número de granjas para apanhe referente ao dia do abate.

\section{Lista Restrita de Candidatos - LCR}

A LRC será formada com base no número de caminhões, ou seja $|L R C|=13$.

Após a designação das equipes às granjas, é feita uma ordenação, em ordem não crescente relativa ao $t v c$ de cada granja e:

1. A quantidade de aves é dividida em dois grupos, onde cada grupo deverá ter mais ou menos a metade da quantidade de aves disponíveis para o abate; os grupos serão formados como segue:

(a) $\mathrm{O} 1^{\circ}$ grupo para as equipes 1 e 2 e;

(b) $\mathrm{O} 2^{\circ}$ grupo para as equipes 3 e 4 .

2. As granjas que fazem parte do $1^{\circ}$ grupo serão ordenadas alternadamente, por equipe, em ordem não crescente do $t v c$ das granjas;

3. O mesmo procedimento é adotado para as granjas do grupo 2;

4. Após o abate das 13 primeiras cargas é feita uma nova LRC com base na disponibilidade dos caminhões, e então é feita uma nova ordenação.

\section{Fase de Busca Local - Solução Final}

Após a geração da Solução Inicial, Sol_Ini, pela fase construtiva do GRASP, é realizada a fase de busca local, onde é feita uma pesquisa na vizinhança da Sol_Ini, com a finalidade de melhorar a solução. Caso essa melhoria seja encontrada, a solução corrente é retornada como resultado.

\section{Algoritmo da Fase de Busca Local}

O algoritmo da fase de busca local é semelhante ao da fase construtiva, com a diferença de que o mesmo parte da Sol_Ini e efetua vários movimentos de trocas na ordem das cargas, até que se satisfaça um critério de parada, por exemplo um número máximo de iterações, ou encontre a solução ótima para o problema.

\section{Vizinhança da Solução Inicial para o Problema de Espera}

Dada uma Solução Inicial, Sol_Ini, portanto uma Vizinhança da Solução Inicial, Viz(Sol_Ini), é gerada através de um movimento de troca entre duas cargas, de maneira que não se viole nenhuma das restrições de viabilidade.

Seja então, uma solução inicial genérica, Sol_ Ini, tal que:

- Sol_Ini $=\left\{\left(\right.\right.$ cam $\left.\left._{c}, \operatorname{grj}_{g}, e q a\right)\right\}, \operatorname{com} c=1$, $\ldots, 13 ;, g=1, \ldots, n g ; e=1, \ldots, 4$. 
Por exemplo, dada a Sol_Ini, abaixo:

- Sol_Ini $=\{(1,1,1),(2,1,1), \quad(10,2,2)$, $(6,1,3),(9,2,3), \ldots,(13,3,4)\}$.

UmaViz(Sol_Ini), pode ser encontrada, pela troca entre dois de seus elementos, por exemplo, troca-se o terceiro elemento, $(10,2,2)$, com o quarto, $(6,1,3)$. Portanto, a nova solução será:

- Sol_Ini $=\{(1,1,1), \quad(2,1,1), \quad(6,1,3)$, $(10,2,2),(9,2,3), \ldots,(13,3,4)\}$.

Após a troca entre os dois elementos de uma solução inicial, calcula-se o valor da função objetivo e, compara este valor com o valor anterior, caso a nova solução, apresente um valor melhor para a função objetivo, ela é mantida e sua vizinhança passa a ser analisada; o procedimento se repete até que o valor ótimo seja alcançado ou se atinja um número máximo de iterações determinado. Então, a melhor solução é retornada como Solução Final.

\section{Resultados e Discussão}

Para verificar o desempenho do programa, foram executadas 30 simulações, das quais três simulações para cada quantidade de granja. As quantidades de granjas variaram, de três até doze; sendo que cada equipe atendia, no máximo três granjas. Em todas as simulações, o algoritmo encontrou a solução ótima, ou satisfatória. A média de tempo de processamento, foi de $0,4 \mathrm{~s}$ para a fase construtiva e de 1,1 s para a fase de busca local.

O programa fornece como relatório final, uma tabela de Programação Diária dos Motoristas. Para análise e comentários dos resultados obtidos pode-se observar na Tabela 4, a programação gerada pelo método heurístico proposto, para uma segunda-feira. Neste dia foram abatidas 85.685 aves, sendo um total de 29 cargas.

A Função Objetivo apresentou um valor de 0,4 min, se comparado com a programação manual, que foi de $39,2 \min ^{6}$, houve uma redução muito significativa.

Observa-se na Tabela 4 que o maior tec foi de 60 min e, ocorreu na $1^{\mathrm{a}}$ carga, no horário das 4 horas, horário este em que a temperatura não prejudica as aves; o menor tec foi de $5 \mathrm{~min}$ e, foi verificado na $7^{\mathrm{a}}$ carga.

Podem-se verificar os horários em que cada equipe de apanha devem iniciar e terminar o carregamento das aves. Estes são os horários em que as equipes iniciarão e terminarão suas atividades. Encontram-se ainda o tempo total de horas trabalhadas e os intervalos de folga.

Por exemplo, a equipe 1, deverá carregar a sua $1^{\mathrm{a}}$ carga às $00 \mathrm{~h} 56 \mathrm{~min}$; para isso deverá sair para viagem pelo menos até às $22 \mathrm{~h} 59 \mathrm{~min}$ do dia anterior; e esta equipe terminará de carregar a última carga às $10 \mathrm{~h} 13 \mathrm{~min}$ (que corresponde a $17^{\mathrm{a}}$ carga de abate), porém, esta equipe estará livre a partir das $12 \mathrm{~h} 12 \mathrm{~min}$. Tendo trabalhado neste dia, $13 \mathrm{~h} 13 \mathrm{~min}$. Observa-se que esta equipe teve um intervalo de folga de 1 hora, intervalo este que ocorreu entre as $07 \mathrm{~h} 04 \mathrm{~min}$ e $08 \mathrm{~h} 04 \mathrm{~min}$, podendo verificar isso, através do htc da $11^{\text {a }}$ carga e o hic da $13^{\mathrm{a}}$ carga, na Tabela 4 , sendo a equipe que mais trabalhou neste dia.Também temos nesta tabela os horários de início e término de carregamento de cada caminhão, bem como os horários de folgas, entre uma carga e outra, com isso pode ser feito um controle das horas trabalhadas por cada caminhão e motorista.

6 Conforme dados da Tabela 4. 


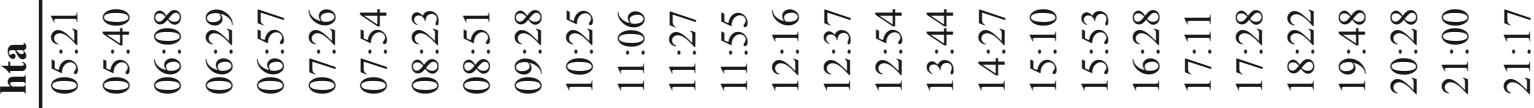

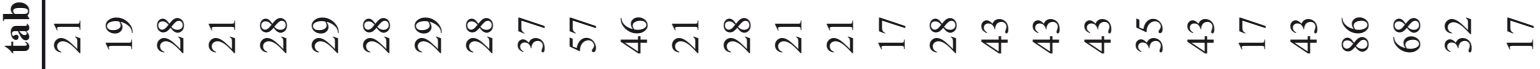

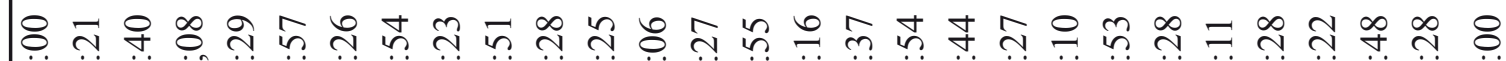

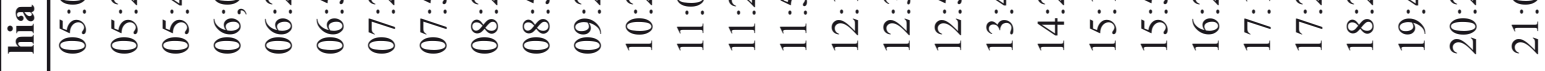

Ð ซ্ৰ

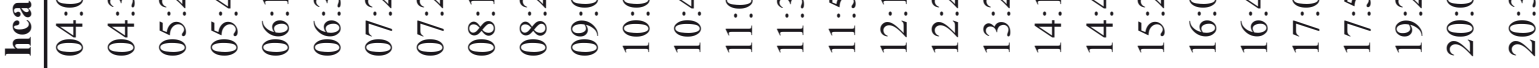

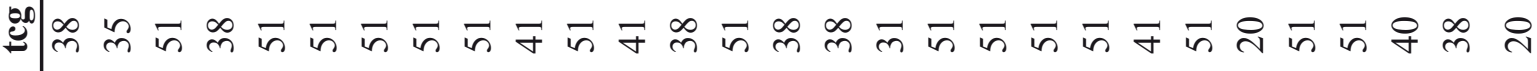
ঋ

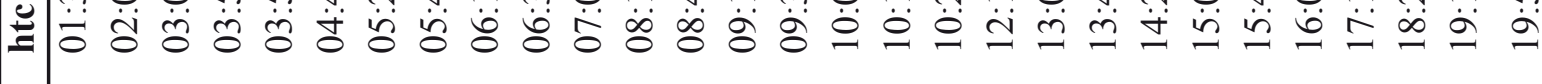

눙

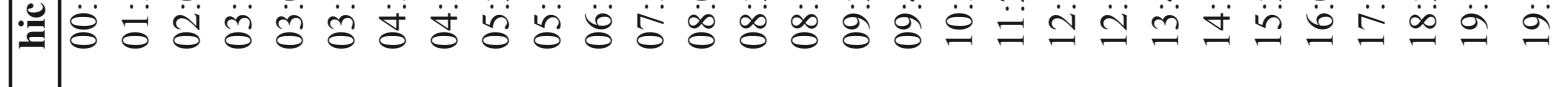

กิ

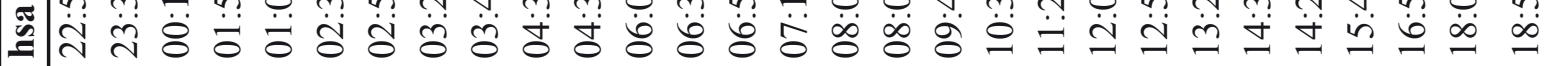

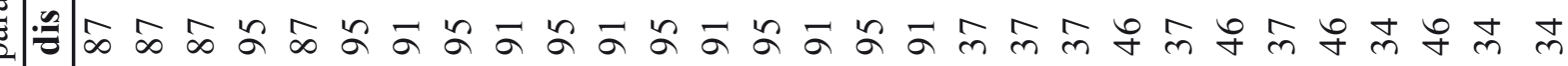

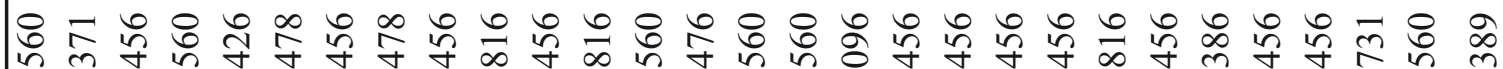
ש্র

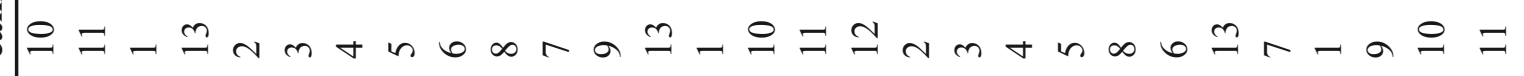

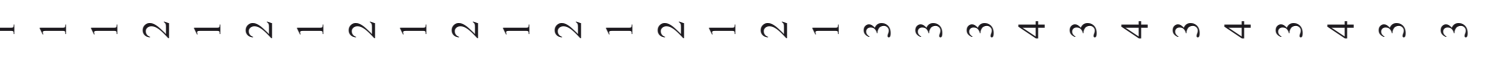

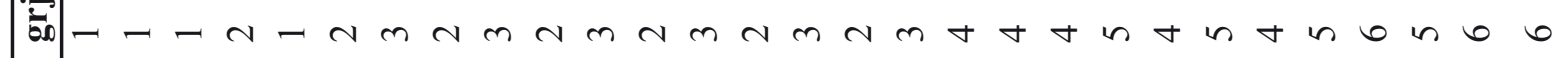
질

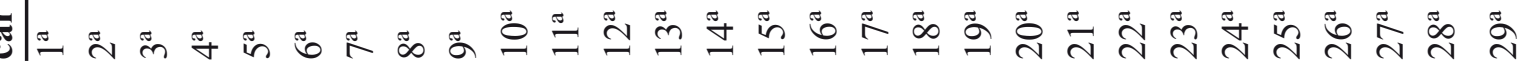

Para a análise do total de horas trabalhadas pelos caminhões, considere os seguintes caminhões: 8 , 1 e 10; o primeiro realizou 2 viagens, e os dois últimos, 3 viagens cada um. Em seguida construiu-se a Tabela 5 com base na Tabela 4. 
Tabela 5 - Número de viagens, horas trabalhadas e intervalos de folgas dos caminhões 1,8 e 10

\begin{tabular}{|c|c|c|c|c|c|c|c|c|c|}
\hline \multirow[t]{2}{*}{ Caminhões } & \multicolumn{2}{|c|}{$1^{a}$ viagem } & \multicolumn{2}{|c|}{$2^{\mathrm{a}}$ viagem } & \multicolumn{2}{|c|}{$3^{\mathrm{a}}$ viagem } & \multirow{2}{*}{$\begin{array}{c}\text { Horas } \\
\text { envolvidas }\end{array}$} & \multirow{2}{*}{$\begin{array}{c}\text { Horas } \\
\text { trabalhadas }\end{array}$} & \multirow{2}{*}{$\begin{array}{l}\text { Horas de } \\
\text { intervalos }\end{array}$} \\
\hline & hsa & hta & hsa & hta & hsa & hta & & & \\
\hline 1 & $00: 12$ & 06:08 & $06: 58$ & $11: 55$ & $15: 48$ & $19: 48$ & $19: 36$ & $14: 53$ & $04: 43$ \\
\hline 8 & $04: 32$ & 09:28 & $12: 54$ & $16: 28$ & - & - & $11: 56$ & $08: 30$ & $03: 26$ \\
\hline 10 & $22: 59$ & $05: 21$ & $07: 19$ & $12: 16$ & $18: 06$ & $21: 00$ & $22: 01$ & $14: 13$ & $07: 48$ \\
\hline
\end{tabular}

Pode-se verificar na Tabela 4 que o caminhão 1 teve envolvido $19 \mathrm{~h} 36 \mathrm{~min}$; o caminhão 10 , 22h01min; o caminhão 8, 11h56min. Descontando os intervalos entre as cargas, verifica-se que o caminhão 1 trabalhou 14 h53min; o caminhão 8 , 08h30min e o caminhão 10, 14h13min.

$\mathrm{Na}$ Tabela 4, na coluna Cam, que se refere aos caminhões, pode-se verificar que a numeração vai do 1 ao 13. Os caminhões numerados do 1 ao 7 , referem-se aos caminhões do tipo I, caminhões com capacidade máxima de 3.456 aves transportadas; os caminhões do 8 ao 9 são caminhões do tipo II caminhões com capacidade máxima de 2.816 aves transportadas; e os caminhões numerados do 10 ao 13 são do tipo III, caminhões com capacidade máxima de 2.560 aves transportadas.

\section{Conclusões}

Com a implementação computacional do método pode-se concluir que todos os agentes envolvidos no processo poderão ter ganhos, como por exemplo:

- Com a diminuição no tempo de espera para o abate, a Avícola e os produtores integrados ganharão, pois haverá uma diminuição da perda de peso e consequente redução da taxa de mortalidade;

- Haverá por parte dos produtores, melhor controle no horário de corte das rações das aves, já que a alimentação das aves deve ser cortada 7 horas antes do abate das mesmas;

- Melhoria no controle da frota por parte da empresa de transporte, pois, a mesma saberá os horários e os locais onde deverão estar cada caminhão, além de ter o controle das equipes de apanha, com relação aos horários de saídas, chegadas e intervalos;

- As equipes de apanha poderão controlar melhor suas atividades;

- Os motoristas poderão controlar melhor suas viagens, pois saberão com antecedência os locais e os horários onde deverão estar.

Para as equipes de apanha, houve uma redução no total de horas trabalhadas nas simulações consideradas. Analisando estes resultados, verificase que a empresa de transporte deverá contratar mais quatro motoristas para satisfazer a restrição do número de horas trabalhadas por cada motorista.

Foram realizadas algumas simulações com três equipes, porém, em todas elas, o número de horas que cada equipe estaria envolvida passava de 15 horas diária, por isso, adotou-se trabalhar com quatro equipes, já que a Avícola tem intenção de aumentar o seu abate diário.

\section{Referências}

AIEX, R. M. Uma investigação experimental da distribuição de probabilidade do tempo de solução em heurísticas GRASP e sua aplicação na análise de implementações paralelas. Rio de Janeiro. 2004. Tese (Doutorado em Ciências-Informática) - Pontifícia Universidade Católica do Rio de Janeiro, Rio de Janeiro.

FEO, T. A.; RESENDE, M. G. C. A probabilistic heuristic for a computacionally difficult set covering problem. Operations Research Letters, Amsterdam, v. 8, p. 67-71, 1989. 
Greedy randomized adative search procedures.

Journal of Global optimization, Dordrecht, v. 6, p. 109133, 1995.

FERNANDES, C. R. Programação de caminhões para $o$ transporte de aves domésticas para o abate via a metaheurística GRASP. 2005. Dissertação (Mestrado em Tecnologia e Ciências Exatas) - Universidade Federal do Paraná, Curitiba.

FESTA, P.; RESENDE, M. G. C. GRASP: an annotated bibliography. In: RIBEIRO, C.; HANSEN, P. (Ed.). Essays and surveys in metaheuristics. Noewell: Kluwer Academic, 2002. p. 325-367.

GOMES, L. M.; DINIZ, V. B.; MARTINHON, C. A. An hybrid GRASP + VNS metaheuristic for the prize-collecting traveling salesman problem. Niterói: Universidade Federal Fluminense, Instituto de Computação, 2000. Work Paper.

HANSEN, P.; MLADENOVIĆ, N. Variable neighborhood search. Computers and Operations Research, New York, v. 24, p. 1097-1100, 1997.

OLIVO, N. Delícias das carnes brancas. 3. ed. Criciúma: Ed. do Autor, 2004.

Recebido em 27 Abril 2011-Received on April 27, 2011.

Aceito em 16 Setembro, 2011 - Accepted on September 16, 2011. 
